\title{
LEARNING THE MORPHOLOGICAL DIVERSITY*
}

\author{
GABRIEL PEYRÉ ${ }^{\dagger}$, JALAL FADILI $^{\ddagger}$, AND JEAN-LUC STARCK ${ }^{\S}$
}

\begin{abstract}
This article proposes a new method for image separation into a linear combination of morphological components. Sparsity in global dictionaries is used to extract the cartoon and oscillating content of the image. Complicated texture patterns are extracted by learning adapted local dictionaries that sparsify patches in the image. These global and local sparsity priors together with the data fidelity define a non-convex energy and the separation is obtained as a stationary point of this energy. This variational optimization is extended to solve more general inverse problems such as inpainting. A new adaptive morphological component analysis algorithm is derived to find a stationary point of the energy. Using adapted dictionaries learned from data allows to circumvent some difficulties faced by fixed dictionaries. Numerical results demonstrate that this adaptivity is indeed crucial to capture complex texture patterns.
\end{abstract}

Key words. Adaptive morphological component analysis, sparsity, image separation, inpainting, dictionary learning, cartoon images, texture, wavelets.

AMS subject classifications. 41A25, 42C40, 65T60

Morphological diversity is a concept where an image is modeled as a sum of components, each of these components having a given morphological signature. Sparsity in a redundant dictionary built by amalgamating several sub-dictionaries can be used to discriminate between these signatures, and fast algorithms such as the Morphological Component Analysis (MCA) have been developed to reconstruct simultaneously all the morphological components [48]. For natural images, containing both edges/contours and oscillatory textures, two fixed dictionaries can be used such as the local DCT for representing the texture and the curvelet for the edges [49]. Results were interesting, but this approach presents some limitations since complicated textures may not be well represented by the local DCT, leading to a poor separation.

This paper extends the morphological diversity concept by learning the morphologies of complicated texture layers to enhance the separation process. These learned dictionaries are coupled with more traditional fixed morphologies to characterize the cartoon and oscillating content of an image. A new adaptive morphological component analysis algorithm performs iteratively and alternately both the learning and the separation.

\section{Image Separation and Inverse Problem Regularization.}

1.1. Image Separation. The image separation process decomposes an input image $u \in \mathbb{R}^{N}$ of $N$ pixels into a linear combination of $|\Lambda|$ layers $\left\{u_{s}\right\}_{s \in \Lambda}$, the socalled morphological components

$$
u=\sum_{s \in \Lambda} u_{s}+\varepsilon
$$

*This work has been done with the support of the French "Agence Nationale de la Recherche" (ANR), under grant NatImages (ANR-08-EMER-009), "Adaptivity for natural images and texture representations".

${ }^{\dagger}$ Ceremade, Université Paris-Dauphine, Place du Maréchal De Lattre De Tassigny, 75775 Paris Cedex 16, (FRANCE) (gabriel.peyre@ceremade.dauphine.fr).

$\ddagger$ GREYC CNRS-ENSICAEN-Université de Caen 6, Bd du Maréchal Juin, 14050 Caen Cedex (FRANCE) (jalal.fadili@greyc.ensicaen.fr)

$\S$ Service d'Astrophysique, CEA/Saclay, Orme des Merisiers, Bat 709, 91191 Gif-sur-Yvette Cedex (FRANCE) (jstarck@cea.fr) 
where $\varepsilon$ is an error term representing noise and model imperfections. Each $u_{s}$ accounts for a different kind of features of the original data $u$.

Image separation is usually achieved by solving a variational optimization problem of the form

$$
\min _{\left\{u_{s}\right\}_{s \in \Lambda}} \frac{1}{2}\left\|u-\sum_{s \in \Lambda} u_{s}\right\|^{2}+\mu \sum_{s \in \Lambda} E_{s}\left(u_{s}\right)
$$

where each energy $E_{s}: \mathbb{R}^{N} \mapsto \mathbb{R}^{+}$favors images with some specific kind of structures. More precisely, for successful separation, each energy $E_{s}$ is designed to be as small as possible over the layer $u_{s}$ it is serving, while being large (or at least not as small) over the other components. Thus each of these layers has its attached prior $E_{s}$, and multiplying the number of priors might help to recover intricate image structures such as smooth areas, edges and textures of natural images.

1.2. Inverse Problems Regularization. Many problems in image processing can be cast as inverting a linear system $f=\mathcal{K} u+\varepsilon$ where $u \in \mathbb{R}^{N}$ is the data to recover, $f \in \mathbb{R}^{m}$ is the observed image and $\varepsilon$ is a Gaussian white noise of known finite variance. The bounded linear operator $\mathcal{K}: \mathbb{R}^{N} \mapsto \mathbb{R}^{m}$ is typically ill-behaved since it models an acquisition process that entails loss of information. This yields ill-posedness of the inverse problem.

This inversion problem is regularized by adding some prior knowledge on the typical structures of the original image $u$. This prior information accounts for the smoothness of the solution and can range from uniform smoothness assumption to more complex knowledge of the geometrical structures of $u$. The decomposition problem (1.2) is extended to handle an ill-behaved operator $\mathcal{K}$ which yields the following minimization problem

$$
\min _{\left\{u_{s}\right\}_{s \in \Lambda}} \frac{1}{2}\left\|f-\mathcal{K} \sum_{s \in \Lambda} u_{s}\right\|^{2}+\mu \sum_{s \in \Lambda} E_{s}\left(u_{s}\right) .
$$

The simple case of image separation (1.1) corresponds to $\mathcal{K}=\operatorname{Id}_{N}$. Typical examples of inverse problems include deconvolution, inpainting and super-resolution. In the latter, one seeks to recover a high-resolution image $u$ from a low-resolution observation $f$. In such a case, $\mathcal{K}$ is the convolution by a blurring kernel followed by a sub-sampling, and $f$ lacks the high frequency content of $u$.

There is a flurry of research activity on linear inverse problems regularization in image processing. Comprehensive overviews can be found in dedicated monographs.

Sparsity-based regularization (e.g. in the wavelet domain) methods have recently received considerable attention, either by adopting a Bayesian expectationmaximization framework [25, 26, 6], by introducing surrogate functionals [14], or using a forward-backward splitting proximal framework [12, 23]. This framework has been successfully applied to inpainting [21, 23], deconvolution [24], multichannel data restoration and blind source separation $[53,7]$.

1.3. Image Inpainting. This paper focuses on the inpainting inverse problem. Inpainting is to restore missing image information based upon the still available (observed) cues from destroyed or deliberately masked subregions of the image $f$.

The inpainting problem corresponds to a diagonal operator

$$
\mathcal{K}=\operatorname{diag}_{i}\left(\eta_{i}\right) \quad \text { where } \quad \eta_{i}=\left\{\begin{array}{lll}
1 & \text { if } & i \notin \Omega, \\
0 & \text { if } & i \in \Omega .
\end{array}\right.
$$


where $\Omega \subset\{0, \ldots, N-1\}$ denotes the set of missing pixels.

Inpainting of non-textured images has been traditionally approached by diffusion equations that progressively fills the missing pixels. The original work of Masnou and Morel makes use of the continuation law of the level sets [35]. Following their work, several authors proposed high order PDEs, see for instance $[10,5,3]$ and anisotropic diffusion [50] for non-texture inpainting. The inpainting of complicated textures can be achieved by copy-and-paste using available information from best matching candidates (e.g. surrounding pixels/regions) assuming self-similarity of the image. This idea underlies many methods in computer graphics [13]. The Morphological Component Analysis (MCA) is able to solve the inpainting problem [21, 23] for images containing simple textural content such as locally parallel oscillations.

\section{Morphological Diversity Modeling with Sparsity.}

2.1. Cartoon Modeling. For the sketchy part of an image, a usual prior is to assume that it belongs to some non-linear Banach space that favors the discontinuities in the image. In particular, this entails that the functional norm of the sketchy part in such spaces is small. Such spaces include the bounded variation (BV) space with the associated total variation norm introduced by Rudin, Osher and Fatemi [46]. Another important prior exploits the sparsity of wavelet coefficients, which corresponds to various kinds of Besov norms. A standard example of such sparsity-promoting prior is the $\ell^{1}$-norm popularized by Donoho and Johnstone [17] in the wavelet context for denoising purposes.

Wavelets are however sub-optimal to efficiently capture edge singularities distributed along smooth curves. The curvelet tight frame, introduced by Candès and Donoho [9], is able to better represent cartoon images with smooth edges. Sparsity in a curvelet tight frame can thus improve the modeling of edges.

2.2. Oscillating Texture Modeling. Simple texture models can also be defined through variational energies that favor oscillations in images. Toward this goal, Meyer introduced a functional space where oscillating patterns have a small norm. It turns out that this Banach space is close to the dual of the BV space [37]. Meyer has defined the so-called G-norm that can be used to perform the decomposition of an image into a cartoon component (using for instance the total variation norm) and an oscillating texture component.

The work of Meyer paved the way to an active research area in variational image processing: cartoon+texture image decomposition. Several authors have proposed algorithms to solve Meyer's problem or close extensions. We note for instance iterative optimization schemes such as the one proposed by Aujol et al. [2]. Other algorithms include $[52,30]$, to cite only a few.

Sparsity-based energies have also been proposed to decompose an image into cartoon+oscillating texture. To this end, Starck and co-authors [21, 49, 48] introduced the MCA framework, where overcomplete fixed dictionaries (one for each layer) are used as a source of diversity to discriminate between the components. The key is that each dictionary must sparsify the corresponding layer while being highly inefficient in representing the other content. For example, MCA is capable of decomposing an image into structure+oscillating texture, using the wavelet or curvelet dictionary for the cartoon layer, and the frame of local cosines for the oscillating texture.

Other dictionaries can enhance over the results of local cosines to capture warped locally oscillatory patterns. For instance, the waveatoms of Demanet and Ying [15] and the brushlets of Meyer and Coifman [36] have been designed for this goal. 
However, the standard MCA is intrinsically limited by the discriminative performance of its fixed non-adaptive dictionaries. Obviously, the latter are not able to sparsify complex textures appearing in natural images.

2.3. Adaptivity and Dictionary Learning. To enhance the modeling of complicated edge layouts and intricate texture patterns, one needs to resort to adapted energies, that are tuned to fit the geometry of complex images.

A class of adaptive methods consists in using a family of orthogonal bases and look for the best basis in this family using combinatorial optimization algorithms. The wedglets [16] and the bandlets [28, 45] better represent contours than a traditional wavelet dictionary. For oscillating textures, a proper basis of the wavelet packet tree [33] with an appropriate tiling of the frequency domain sparsifies some oscillatory patterns. Cosine packets allow a dyadic partition of the spatial domain [33] according to a quad-tree structure. Grouplet bases [34] are able to approximate efficiently oscillating and turbulent textures, and were successfully applied to texture synthesis and inpainting in [42].

Contrary to these approaches, which are able to handle only a particular kind of images or textures, other approaches can adapt to the content of images through a learning process. By minimizing a sparsity criterion, such algorithms allow to optimize a local dictionary for a set of exemplar patches.

Olshausen and Field [40] were the first to propose a way of learning the dictionary from the data and to insist on the dictionary redundancy. They have applied this learning scheme to patches extracted from natural images. The major conclusion of this line of research is that learning over a large set of disparate natural images leads to localized oriented edge filters. Since then, other approaches to sparse coding have been proposed using independent components analysis [4], or different sparsity priors on the representation coefficients $[29,27,22,1]$.

It is worth point out that this dictionary learning bears tight similarities with sparsity-based blind source separation (BSS) algorithms as proposed in [53] and in the GMCA algorithm [7]. The role played by the dictionary parallels the one of the mixing matrix in BSS.

These learned dictionaries have proven useful to perform image denoising [19], inpainting [32], texture synthesis [43] and image recognition [31].

A preliminary description of the adaptive MCA method was presented in [44]. Shoham and Elad have developed in [47] an adaptive separation method that approximately minimizes our adaptive MCA energy, that is faster if no global dictionary is used.

2.4. Contributions. This paper proposes a new adaptive image separation method. It extends previous work on morphological component analysis by adapting the dictionary used to model and discriminate complex texture layer(s). Section 3 introduces the notions of global and local dictionaries, that can be combined to achieve high quality separation. The local dictionaries are learned during the separation within a new adaptive morphological component analysis algorithm detailed in Section 4. This algorithm converges to a stationary point of a non-convex variational energy. Numerical results show that a single adaptive texture layer can be trained without user intervention to extract a complicated texture. Additional layers can be added as well, and in this case, a proper user-defined initialization is required to drive the algorithm to the desired decomposition which corresponds to a particular stationary point of the energy. This option offers some flexibility to the user to guide the decomposition algorithm toward the desired solution. 
3. Global and Local Sparsity Energies. Each energy $E_{s}\left(u_{s}\right)$ depends on a dictionary $D_{s}$ that is a collection of atoms used to describe the component $u_{s}$ sparsely. This paper considers both global dictionaries, that are used to describe the content of the component as a whole, and local dictionaries that are applied to (local) patches extracted from the component.

The set of indices is thus decomposed as $\Lambda=\Lambda^{\mathrm{G}} \cup \Lambda^{\mathrm{L}}$, and to each global layer $s \in \Lambda^{\mathrm{G}}$ is assigned a global energy $E^{\mathrm{G}}\left(u_{s}, D_{s}\right)$, whereas a local layer uses a local energy $E^{\mathrm{L}}\left(u_{s}, D_{s}\right)$. The global dictionaries are fixed by the user and correspond typically to a cartoon morphological component or simple oscillating patterns. On the contrary, local dictionaries $\left\{D_{s}\right\}_{s \in \Lambda^{\mathrm{L}}}$ are learned by our algorithm to capture complicated stationary texture pattern. The corresponding adaptive separation process thus extends (1.2) to an optimization on both the components and the local dictionaries

$$
\min _{\left\{u_{s}\right\}_{s \in \Lambda},\left\{D_{s} \in \mathcal{D}_{s}\right\}_{s \in \Lambda^{\mathrm{L}}}} \frac{1}{2}\left\|f-\mathcal{K} \sum_{s \in \Lambda} u_{s}\right\|^{2}+\mu \sum_{s \in \Lambda^{\mathrm{G}}} E^{\mathrm{G}}\left(u_{s}, D_{s}\right)+\mu \sum_{s \in \Lambda^{\mathrm{L}}} E^{\mathrm{L}}\left(u_{s}, D_{s}\right),
$$

where $\mathcal{D}_{s}$ is a suitable set of convex constraints.

3.1. Sparsity-based Energy for a Global Dictionary. A global dictionary $D_{s}=\left(d_{s, j}\right)_{0 \leqslant j<m_{s}}$, for $s \in \Lambda^{\mathrm{G}}$, is a (possibly redundant) collection of $m_{s} \geqslant N$ atoms $d_{s, j} \in \mathbb{R}^{N}$, that can be represented as a rectangular matrix $D_{s} \in \mathbb{R}^{N \times m_{s}}$. The decomposition of a component $u_{s}$ using this dictionary reads

$$
u_{s}=D_{s} x_{s}=\sum_{j=0}^{m_{s}-1} x_{s}[j] d_{s, j} .
$$

For a redundant dictionary where $m_{s}>N$, such a decomposition is non-unique, and a sparsity-promoting energy favors sparse coefficients $x_{s}$, for which most of the entries $x_{s}[j]$ are zero. In this paper we use a convex $\ell^{1}$ sparsity measure

$$
\left\|x_{s}\right\|_{1}=\sum_{j=0}^{m_{s}-1}\left|x_{s}[j]\right|,
$$

which was introduced by Chen, Donoho and Saunders [11] in the basis pursuit denoising method for sparse approximation.

Finding a sparse approximation $D_{s} x_{s}$ of $u_{s}$ in $D_{s}$ can then be formulated as minimizing the following global energy

$$
\begin{gathered}
E^{\mathrm{G}}\left(u_{s}, D_{s}\right)=\min _{x_{s} \in \mathbb{R}^{m_{s}}} \mathcal{E}^{\mathrm{G}}\left(u_{s}, x_{s}, D_{s}\right), \\
\text { where } \mathcal{E}^{\mathrm{G}}\left(u_{s}, x_{s}, D_{s}\right)=\frac{1}{2}\left\|u_{s}-D_{s} x_{s}\right\|^{2}+\lambda\left\|x_{s}\right\|_{1} .
\end{gathered}
$$

The parameter $\lambda$ allows an approximate reconstruction $D_{s} x_{s} \approx u_{s}$ and should be adapted to the noise level $\|\varepsilon\|$.

Cartoon sparse models. Wavelets [33] are used extensively in image compression and allow to capture efficiently images with isotropic singularities and images with bounded variations. We use a redundant dictionary $D_{\text {wav }}$ of translation invariant wavelets to capture the sketchy content of an image, which is assumed to have a small total variation.

To capture more regular edge patterns, we use a redundant tight frame of curvelets $D_{\text {curv }}$, introduced by Candès and Donoho [9] to represent optimally cartoon images with $\mathrm{C}^{2}$-regular edge curves. 
Oscillating sparse models. Locally oscillating and stationary textures are handled with a redundant tight frame $D_{\text {dct }}$ of local cosines [33]. We use local cosine atoms defined on patches of $32 \times 32$ pixels, with an overlapping factor of 2 along the horizontal and vertical directions, so that the redundancy of $D_{\text {dct }}$ is $m_{s} / N=4$. As explained in the introduction, other dictionaries well-suited for sparsifying oscillating patterns could be used as well, e.g. waveatoms [15].

3.2. Sparsity-based Energy for a Local Dictionary. We use local dictionaries $\left\{D_{s}\right\}_{s \in \Lambda^{\mathrm{L}}}$ to capture fine scale structures of the textures. For $s \in \Lambda^{\mathrm{L}}$, a local dictionary $D_{s} \in \mathbb{R}^{n \times m_{s}}$ is used to represent patches $R_{k}\left(u_{s}\right) \in \mathbb{R}^{n}$ of $n=\tau \times \tau$ pixels extracted from a component $u_{s}$,

$$
\forall 0 \leqslant k_{1}, k_{2}<\frac{\sqrt{N}}{\Delta},-\tau / 2 \leqslant i_{1}, i_{2}<\tau / 2, \quad R_{k}\left(u_{s}\right)[i]=u_{s}\left(k_{1} \Delta+i_{1}, k_{2} \Delta+i_{2}\right),
$$

where $i=\left(i_{1}, i_{2}\right)$ is the location of a pixel in the patch, $k=\left(k_{1}, k_{2}\right)$ indexes the patch location and is represented as an integer in $\left\{0, \ldots, N / \Delta^{2}-1\right\}$, and $1 \leqslant \Delta \leqslant \tau$ controls the sub-sampling of the patch extraction process.

Similarly to the energy (3.2) associated to a global dictionary, we define an energy $E^{\mathrm{L}}\left(u_{s}, D_{s}\right)$ associated to a local dictionary $D_{s}$. This energy allows one to control the sparsity of the decomposition of all the patches $R_{k}\left(u_{s}\right)$ in $D_{s}$. Following Aharon and Elad $[19,1]$, we define this energy $E^{\mathrm{L}}\left(u_{s}, D_{s}\right)$ as

$$
E^{\mathrm{L}}\left(u_{s}, D_{s}\right)=\min _{\left\{x_{s, k}\right\}_{k} \in \mathbb{R}^{m_{s} \times N / \Delta^{2}}}, \mathcal{E}^{\mathrm{L}}\left(u_{s},\left\{x_{s, k}\right\}_{k}, D_{s}\right)
$$

$$
\text { where } \mathcal{E}^{\mathrm{L}}\left(u_{s},\left\{x_{s, k}\right\}_{k}, D_{s}\right)=\frac{1}{p} \sum_{k}\left(\frac{1}{2}\left\|R_{k}\left(u_{s}\right)-D_{s} x_{s, k}\right\|^{2}+\lambda\left\|x_{s, k}\right\|_{1}\right) \text {, }
$$

where $p=(\tau / \Delta)^{2}=n / \Delta^{2}$. Each $x_{s, k}$ corresponds to the coefficients of the decomposition of the patch $R_{k}\left(u_{s}\right)$ in the dictionary $D_{s}$. The weight $1 / p$ in the energy (3.4) compensates for the redundancy factor introduced by the overlap between the patches $R_{k}\left(u_{s}\right)$. This normalization allows one to re-scale the local energy (3.4) to be comparable with the global one (3.2).

3.3. Images vs. Coefficients. The variational formulation (3.1) proposed in this paper directly seeks for the components $\left\{u_{s}\right\}_{s \in \Lambda}$, and the coefficients are only considered as auxiliary variables. Alternative formulations of inverse problems in redundant dictionaries or union of bases would look instead for the coefficients $x_{s}$ or $\left\{x_{s, k}\right\}_{k}$ of each component in the dictionary $D_{s}$.

The corresponding coefficient-based minimization reads

$$
\begin{gathered}
\left(\left\{x_{s}^{\star}\right\}_{s \in \Lambda},\left\{D_{s}^{\star}\right\}_{s \in \Lambda^{\mathrm{L}}}\right) \in \underset{\left\{x_{s}\right\}_{s \in \Lambda},\left\{D_{s} \in \mathcal{D}_{s}\right\}_{s \in \Lambda^{\mathrm{L}}}}{\operatorname{argmin}} \\
\frac{1}{2}\left\|f-\mathcal{K} \sum_{s \in \Lambda^{\mathrm{G}}} D_{s} x_{s}-\mathcal{K} \sum_{s \in \Lambda^{\mathrm{L}}, k} R_{k}^{*}\left(D_{s} x_{s, k}\right)\right\|^{2}+\lambda \sum_{s \in \Lambda^{\mathrm{G}}}\left\|x_{s}\right\|_{1}+\lambda \sum_{s \in \Lambda^{\mathrm{L}}, k}\left\|x_{s, k}\right\|_{1},
\end{gathered}
$$

where the dual operator $R_{k}^{*}$ reconstructs an image in $\mathbb{R}^{N}$ with zero values outside the patch. Such a coefficient-based optimization is used for global dictionaries in $[49,21,23]$. 
A global components for $s \in \Lambda^{\mathrm{G}}$ is retrieved from these optimized coefficients as $u_{s}^{\star}=D_{s} x_{s}^{\star}$. For a local dictionary, the reconstruction from the patches are averaged

$$
\forall s \in \Lambda^{\mathrm{L}}, \quad u_{s}^{\star}=\sum_{k} R_{k}^{*}\left(D_{s}^{\star} x_{s, k}^{\star}\right) .
$$

The reconstruction formula (3.7) shows that the optimization (3.6) for the local component $s \in \Lambda^{\mathrm{L}}$ corresponds to finding a sparse approximation of $u_{s}$ in the highly redundant global dictionary $\left\{R_{k}^{*}\left(d_{s, j}\right)\right\}_{k, j}$ that gathers all the atoms at all patch locations.

The two formulations (3.1) and (3.6) are expected to differ significantly. These differences share some similarities to the one analyzed in [20], that studies analysis and synthesis signal priors. In our setting, where we use local dictionaries, the formulation (3.1) over the image domain makes more sense. In this formulation, each patch is analyzed independently by the sparsity prior, and the $L^{2}$ fidelity term gathers linearly the contributions of the patches to obtain $u_{s}$. As noticed by Aharon and Elad $[19,1]$, this average of sparse representations has some flavor of minimum mean square estimation, which further helps to reduce the noise.

Furthermore, the formulation (3.6) corresponds to the optimization of coefficients in a highly redundant dictionary, which is demanding numerically. In contrast, our formulation (3.1) allows for an iterative scheme that optimizes the coefficients independently over each patch and average them afterward. We describe this adaptive MCA scheme in the following section.

The last chief advantage of (3.1) is that it decouples the contribution of each local dictionary $D_{s}$, for $s \in \Lambda^{\mathrm{L}}$. This simplifies the learning process, since each dictionary is independently optimized during the iterations of our adaptive MCA.

4. Adaptive Morphological Component Analysis. The morphological component analysis (MCA) algorithm [49, 48] allows to solve iteratively the variational separation problem (1.3) for sparsity-based energies $E_{s}$ as defined in (3.2). For the decomposition of an image into its geometrical and textured parts, the original approach $[49,48]$ uses fixed dictionaries of wavelets $D_{\text {wav }}$, curvelets $D_{\text {curv }}$, and local cosines $D_{\text {dct }}$. This paper extends the MCA algorithm to deal with energies $E_{s}$ associated to local dictionaries $D_{s}$ as defined in (3.4). In addition, our adaptive MCA algorithm is able to optimize the local dictionaries $D_{s}$, which are automatically adapted to the texture to extract.

4.1. Adaptive Variational Problem. The new adaptive MCA algorithm minimizes iteratively the energy (1.3) by adding to the decomposition variables $\left\{u_{s}\right\}_{s \in \Lambda}$ and $\left\{D_{s}\right\}_{s \in \Lambda^{\mathrm{L}}}$ auxiliary variables $\left\{x_{s}\right\}_{s \in \Lambda}$ corresponding to the coefficients of the decomposition of each $u_{s}$. For a global layer $s \in \Lambda^{\mathrm{G}}$, these coefficients are stored in a vector $x_{s} \in \mathbb{R}^{m_{s}}$. For a local dictionary $s \in \Lambda^{\mathrm{L}}$ these coefficients are a collection of vectors $\left\{x_{s, k}\right\}_{k=0}^{N / \Delta^{2}-1} \in \mathbb{R}^{m_{s} \times N / \Delta^{2}}$.

The energy minimized by the adaptive MCA algorithm is

$$
\begin{aligned}
& \mathcal{E}\left(\left\{u_{s}\right\}_{s},\left\{x_{s}\right\}_{s},\left\{D_{s} \in \mathcal{D}_{s}\right\}_{s \in \Lambda^{\mathrm{L}}}\right)=\frac{1}{2}\left\|f-\mathcal{K} \sum_{s} u_{s}\right\|^{2}+ \\
& \mu \sum_{s \in \Lambda^{\mathrm{G}}} \mathcal{E}^{\mathrm{G}}\left(u_{s}, x_{s}, D_{s}\right)+\mu \sum_{s \in \Lambda^{\mathrm{L}}} \mathcal{E}^{\mathrm{L}}\left(u_{s}, x_{s}, D_{s}\right)
\end{aligned}
$$

where the global and local energies $\mathcal{E}^{\mathrm{G}}$ and $\mathcal{E}^{\mathrm{L}}$ are defined in (3.3) and (3.5). 
The constraint $D_{s}=\left(d_{j, s}\right)_{0 \leqslant j<m_{s}} \in \mathcal{D}_{s}$ for $s \in \Lambda^{\mathrm{L}}$ ensures that the columns $d_{i, s}$ of $D_{s}$ have bounded norm, and avoids the classical scale indeterminacy between the dictionaries and the coefficients. We also impose that the atoms have zero mean, which is consistent with the intuition that a texture contains locally only high frequencies. The convex set $\mathcal{D}_{s}$ reads

$$
\mathcal{D}_{s}=\left\{D \in \mathbb{R}^{n \times m_{s}} \backslash \forall j=0, \ldots, m_{s}-1,\left\|d_{j, s}\right\| \leqslant 1 \text { and } \sum_{i} d_{j, s}[i]=0\right\} .
$$

Adaptive non-convex minimization. The energy $\mathcal{E}$ is marginally convex in each of its arguments, and is optimized over a convex set. However, $\mathcal{E}$ is non-convex jointly in all its arguments. We thus propose an iterative block relaxation coordinate descent minimization scheme, and show that it converges to a stationary point of the energy.

The adaptive MCA algorithm operates by minimizing successively $\mathcal{E}$ on the set of components $\left\{u_{s}\right\}_{s \in \Lambda}$, on the set of coefficients $\left\{x_{s}\right\}_{s \in \Lambda}$ and the set of local dictionary $\left\{D_{s}\right\}_{s \in \Lambda^{\mathrm{L}}}$. Each minimization is performed while keeping all remaining variables fixed.

The initialization of the dictionaries $\left\{D_{s}\right\}_{s \in \Lambda^{\mathrm{L}}}$ is thus important, and user intervention can improve the result by selecting initial features relevant for texture extraction.

4.2. Step 1 - Update of the Coefficients $\left\{x_{s}\right\}_{s \in \Lambda}$. The update of the coefficients requires the minimization of $\mathcal{E}$ with respect to $\left\{x_{s}\right\}_{s \in \Lambda}$. Since this problem is separable in each of the coefficient variable $x_{s}$, we perform the optimization independently for the coefficients of each global layer or each patch in a local layer.

For a global dictionary $s \in \Lambda^{\mathrm{G}}$, this corresponds to solving

$$
x_{s}=\underset{x \in \mathbb{R}^{m_{s}}}{\operatorname{argmin}} \frac{1}{2}\left\|u_{s}-D_{s} x\right\|^{2}+\lambda\|x\|_{1} .
$$

For a local dictionary $s \in \Lambda^{\mathrm{L}}$, the minimization is performed with respect to each patch index $k$

$$
x_{s, k}=\underset{x \in \mathbb{R}^{m_{s}}}{\operatorname{argmin}} \frac{1}{2}\left\|R_{k}\left(u_{s}\right)-D_{s} x\right\|^{2}+p \lambda\|x\|_{1} .
$$

Both (4.3) and (4.4) correspond to sparse coding by minimizing a basis pursuit denoising (BPDN or Lasso for statisticians) problem [11]. Various algorithms have been proposed to solve this convex problem efficiently, among which interior point solvers [11], iterative soft thresholding $[14,12]$, or Nesterov multi-steps scheme [38, 39].

4.3. Step 2 - Update of the Components $\left\{u_{s}\right\}_{s \in \Lambda}$. Updating the components $\left\{u_{s}\right\}_{s \in \Lambda}$ requires to solve a quadratic minimization problem

$$
\min _{\left\{u_{s}\right\}_{s \in \Lambda}}\left\|f-\mathcal{K} \sum_{s \in \Lambda} u_{s}\right\|^{2}+\mu \sum_{s \in \Lambda^{\mathrm{G}}}\left\|u_{s}-D_{s} x_{s}\right\|^{2}+\frac{\mu}{p} \sum_{s \in \Lambda^{\mathrm{L}}, k}\left\|R_{k}\left(u_{s}\right)-D_{s} x_{s, k}\right\|^{2} .
$$

This is a high dimensional problem since it involves all the layers, and it can be solved with a conjugate gradient descent.

An alternate method consists in cycling repeatedly on each component $u_{s}$ for $s \in \Lambda$, and optimizing (4.5) with respect to $u_{s}$ alone. This generates iterates $u_{s}^{(\ell)}$ that ultimately converge to a minimizer of (4.5). Although the convergence is slower than 
with a conjugate gradient descent, it is simpler to implement since each component update is easy to compute.

At a step $\ell$ of this update of the components, a new iterate $u_{s}^{(\ell+1)}$ is obtained by minimizing (4.5) with respect to $u_{s}$ alone. For a global dictionary $s \in \Lambda^{\mathrm{G}}$, this leads to

$$
u_{s}^{(\ell+1)}=\underset{u \in \mathbb{R}^{N}}{\operatorname{argmin}}\left\|r_{s}-\mathcal{K} u\right\|^{2}+\mu\left\|u-D_{s} x_{s}\right\|^{2} \quad \text { with } \quad r_{s}=f-\mathcal{K} \sum_{s^{\prime} \neq s} u_{s^{\prime}}^{(\ell)}
$$

and for a local dictionary $s \in \Lambda^{\mathrm{L}}$

$$
u_{s}^{(\ell+1)}=\underset{u \in \mathbb{R}^{N}}{\operatorname{argmin}}\left\|r_{s}-\mathcal{K} u\right\|^{2}+\mu \sum_{k}\left\|R_{k}(u)-D_{s} x_{s, k}\right\|^{2}
$$

This leads to the following update rule

$$
u_{s}^{(\ell+1)}=\left(\mathcal{K}^{*} \mathcal{K}+\mu \operatorname{Id}_{N}\right)^{-1}\left(\mathcal{K}^{*} r_{s}+\mu \hat{u}_{s}\right)
$$

where the reconstructed $\hat{u}_{s}$ is computed differently depending whether the dictionary is global or local

$$
\hat{u}_{s}= \begin{cases}D_{s} x_{s}, & \text { if } \quad s \in \Lambda^{\mathrm{G}}, \\ \frac{1}{p} \sum_{k} R_{k}^{*}\left(D_{s} x_{s, k}\right), & \text { if } \quad s \in \Lambda^{\mathrm{L}} .\end{cases}
$$

To derive the expression for a local dictionary, we used the fact that

$$
\frac{1}{p} \sum_{k} R_{k}^{*} R_{k}=\mathrm{Id}_{N}
$$

and a special care should be taken at the boundaries of the image.

For a general operator $\mathcal{K}$, the update (4.6) requires to solve a well-conditioned linear system, which can be computed by conjugate gradient. If the operator $\mathcal{K}$ is known to be diagonalized in some basis (e.g. Fourier for convolution), this inversion can be computed very efficiently in closed-form. For instance, in the image separation problem, where $\mathcal{K}=\operatorname{Id}_{N}$, the update of the component $u_{s}$ reduces to the convex sum

$$
u_{s}^{(\ell+1)}=(1+\mu)^{-1}\left(r_{s}+\mu \hat{u}_{s}\right) .
$$

In the inpainting problem, the update becomes

$$
u_{s}^{(\ell+1)}[i]= \begin{cases}(1+\mu)^{-1}\left(r_{s}[i]+\mu \hat{u}_{s}[i]\right) & \text { if } i \in \Omega, \\ \hat{u}_{s}[i] & \text { if } i \notin \Omega .\end{cases}
$$

4.4. Step 3 - Update of the Dictionaries $\left\{D_{s}\right\}_{s \in \Lambda^{\mathrm{L}}}$. This update step concerns only local dictionaries $D_{s}$ for $s \in \Lambda^{\mathrm{L}}$. Since this problem is separable in each of the dictionary, this optimization is performed for each $D_{s}$ independently. This corresponds to

$$
D_{s}=\underset{D \in \mathcal{D}_{s}}{\operatorname{argmin}} \sum_{k}\left\|R_{k}\left(u_{s}\right)-D x_{s, k}\right\|^{2}=\underset{D \in \mathcal{D}_{s}}{\operatorname{argmin}}\left\|U_{s}-D X_{s}\right\|_{F}^{2}
$$


where $\|\cdot\|_{F}$ stands for the Forbenius norm, the convex set $\mathcal{D}_{s}$ is defined in (4.2), $U_{s} \in \mathbb{R}^{n \times N / \Delta^{2}}$ is the matrix whose $k$-column is $R_{k}\left(u_{s}\right) \in \mathbb{R}^{n}$, and $X_{s} \in \mathbb{R}^{m_{s} \times N / \Delta^{2}}$ is the matrix whose $k$-column is $x_{s, k} \in \mathbb{R}^{m_{s}}$.

We perform this minimization using a projected gradient descent, that computes iterates $D_{s}^{(\ell)}$

$$
D_{s}^{(\ell+1)}=\mathcal{P}_{\mathcal{D}_{s}}\left(D_{s}^{(\ell)}+\tau\left(U_{s}-D_{s}^{(\ell)} X_{s}\right) X_{s}^{*}\right)
$$

where $\tau<2 /\left\|X_{s} X_{s}^{*}\right\|$ is the step size, and

$$
\left(\tilde{d}_{j}\right)_{j=0}^{m_{s}-1}=\mathcal{P}_{\mathcal{D}_{s}}(D)
$$

is the projection of $D=\left(d_{j}\right)_{j=0}^{m_{s}-1}$ on the convex set $\mathcal{D}_{s}$, that has an explicit expression

$$
\tilde{d}_{j}=\frac{d_{j}-c}{\left\|d_{j}-c\right\|} \quad \text { with } \quad c=\frac{1}{n} \sum_{i=0}^{n-1} d_{j}[i] .
$$

We note that more efficient schemes, such as Nesterov multi-step descent [38, 39], could be used to minimize (4.8). Other approaches, potentially faster, has been used to perform the update of the dictionary $[22,1]$, but they do not minimize exactly (4.8).

Figure 4.1 shows two example of dictionary learned from input exemplar textures. One can see that the learned atoms $d_{j}$ do a good job at capturing the patterns of the exemplar texture.

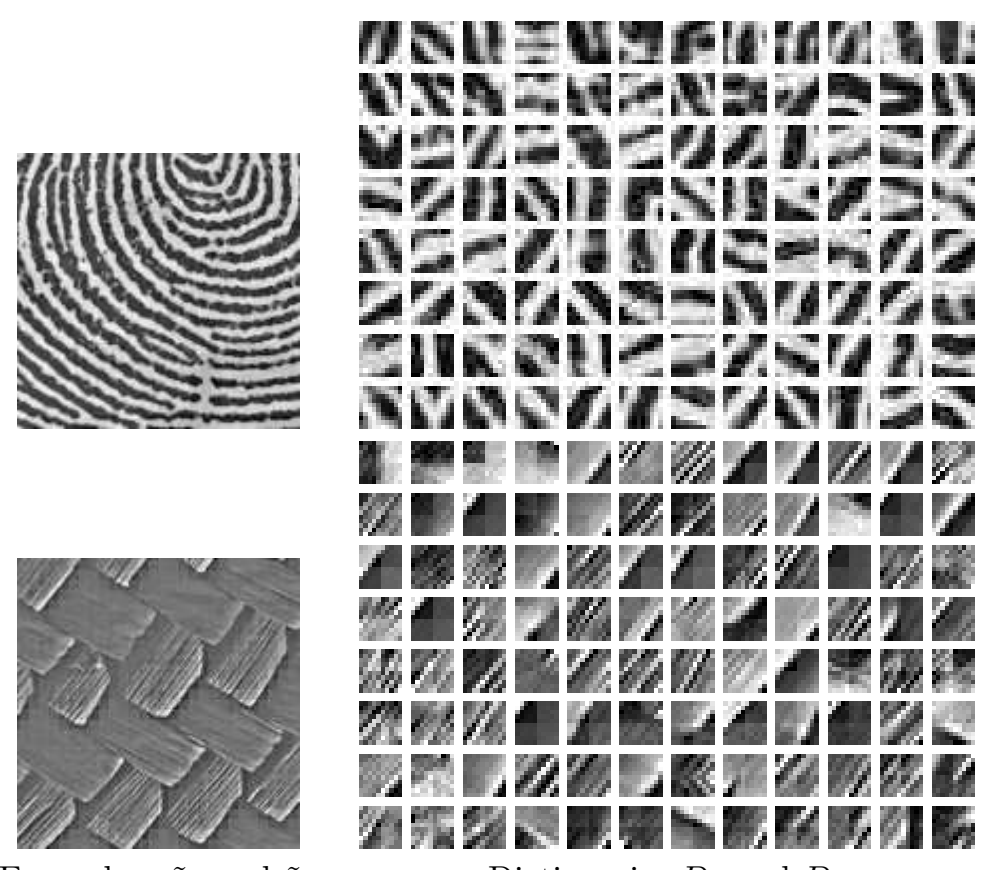

Exemplars $\tilde{u}_{1}$ and $\tilde{u}_{2}$

Dictionaries $D_{1}$ and $D_{2}$

FIG. 4.1. Example of dictionary learning, for patches of size $\tau=10$ pixels (only a subset of the atoms is displayed on the right). 
4.5. Adaptive MCA algorithm. The adaptive MCA algorithm is summarized in Algorithm 1. It iteratively cycles between these three steps. Each step is performed with an iterative algorithm, and a tolerance $\eta_{\text {coef }}, \eta_{\text {comp }}$ and $\eta_{\text {dico }}$ is associate to these inner iterations.

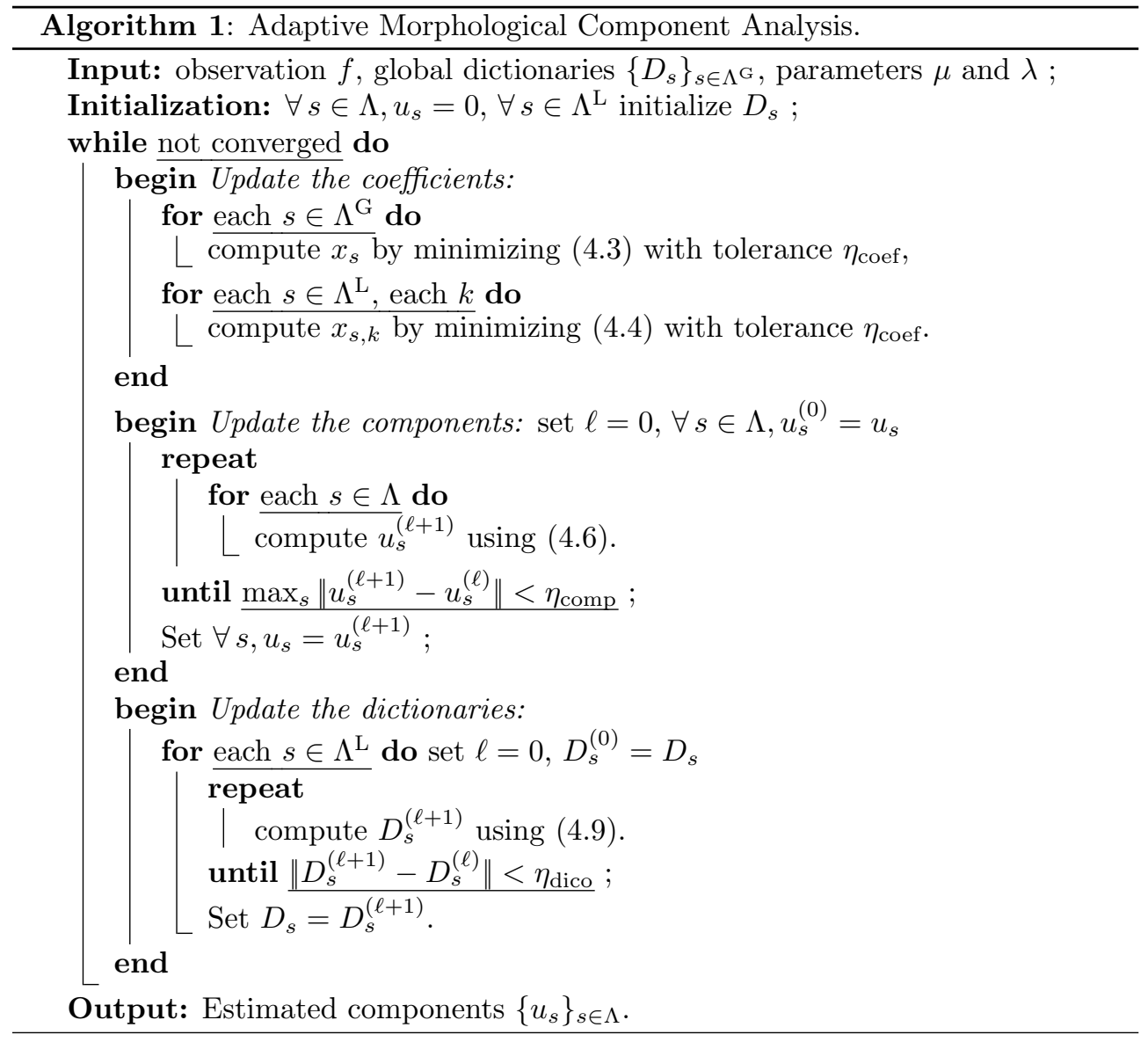

Initialization of the dictionaries. Since the energy $\mathcal{E}$ minimized by the adaptive MCA is non-convex, different initializations for the dictionaries $\left\{D_{s}\right\}_{s \in \Lambda^{\mathrm{L}}}$ might lead to different solutions. In the numerical experiments detailed in Section 5, we consider several initialization scenario, that might require some user intervention.

Convergence of adaptive $M C A$. The following result ensures that this adaptive MCA algorithm converges to a stationary point of the energy $\mathcal{E}$.

Proposition 4.1. Suppose that each of steps 1-3 is solved exactly by the adaptive $M C A$ algorithm. Then, the obtained sequence of iterates is defined, bounded and every accumulation point is a stationary point of $\mathcal{E}$.

Proof. The optimization problem (4.1) has at least one solution by coercivity. The convergence proof of the block-relaxation minimization scheme follows, after identifying our problem with the one considered by the author in [51]. Indeed using a comparable notation to that of [51], we can write $\mathcal{E}\left(\left\{u_{s}\right\}_{s},\left\{x_{s}\right\}_{s},\left\{D_{s}\right\}_{s \in \Lambda^{\mathrm{L}}}\right)$ as

$$
J_{0}\left(\left\{D_{s}\right\}_{s},\left\{u_{s}\right\}_{s},\left\{x_{s}\right\}_{s}\right)+J_{D}\left(\left\{D_{s}\right\}_{s \in \Lambda^{\mathrm{L}}}\right)+J_{u}\left(\left\{u_{s}\right\}_{s}\right)+\sum_{s} J_{x}^{s}\left(x_{s}\right)
$$


where

$$
\begin{aligned}
J_{0}\left(\left\{D_{s}\right\}_{s},\left\{u_{s}\right\}_{s},\left\{x_{s}\right\}_{s}\right) & =\frac{\mu}{2} \sum_{s \in \Lambda^{\mathrm{G}}}\left\|u_{s}-D_{s} x_{s}\right\|^{2}+\frac{\mu}{2} \sum_{s \in \Lambda^{\mathrm{L}}, k}\left\|R_{k}\left(u_{s}\right)-D_{s} x_{s, k}\right\|^{2} \\
J_{D}\left(\left\{D_{s}\right\}_{s}\right) & =\left\{\begin{array}{ll}
0 & \text { if } D_{s} \in \mathcal{D}_{s} \forall s \in \Lambda^{\mathrm{L}}, \\
+\infty & \text { if } D_{s} \notin \mathcal{D}_{s} \text { for some } s \in \Lambda^{\mathrm{L}} .
\end{array},\right. \\
J_{u}\left(\left\{u_{s}\right\}_{s}\right) & =\frac{1}{2}\left\|f-\mathcal{K} \sum_{s} u_{s}\right\|^{2}, \\
J_{x}^{s}\left(x_{s}\right) & =\left\{\begin{array}{ll}
\mu \lambda\left\|x_{s}\right\|_{1}, & \text { (global dictionary), } \\
\mu \lambda / p \sum_{k}\left\|x_{s, k}\right\|_{1}, & \text { (local dictionary). }
\end{array}, \forall s \in \Lambda .\right.
\end{aligned}
$$

It is not difficult to see that $J_{0}$ has a non-empty open domain and is continuously differentiable on its domain. Thus $J_{0}$ satisfies Assumption A.1 in [51]. Moreover, $\mathcal{E}$ is continuous on its effective domain, with bounded level sets. $\mathcal{E}$ is also convex in $\left(u_{1}, \ldots, u_{|\Lambda|}, x_{1}, \ldots, x_{|\Lambda|}\right)$, and $J_{D}$ is convex. Thus, Lemma 3.1 and Theorem 4.1(a) of [51] imply that the sequence of iterates provided by the block-relaxation MCA algorithm is defined, bounded and every accumulation point is a stationary point of $\mathcal{E}$.

It is important to note that the convergence is only guaranteed for an exact adaptive MCA that performs an exact coordinate-wise minimization at each of the three steps. Little is known about the behavior of an approximate block coordinate descent, and the tolerances $\eta_{\text {coef }}, \eta_{\text {comp }}$ and $\eta_{\text {dico }}$ should be decayed through the iterations of the MCA to ensure convergence. For the numerical experiments of Section 5, we use fixed tolerances, which did not deteriorate the visual quality of the results.

Varying threshold. An important feature of the morphological component analysis is that the value of the parameter $\lambda$ is decreased through iterations until it reaches its final value that is adapted to the noise level. This allows to speed up the convergence, and bears similarities with continuation and path following methods for solving BPDN $[18,41]$. More sophisticated threshold update schedules might be used, see for instance $[8]$.

Computational complexity. The bulk of computation in Step 1 of Algorithm 1 is invested in the application of the matrix $D_{s}$ and its adjoint $D_{s}^{*}$. For global dictionaries corresponding to tight frames used in this paper, these matrices are never explicitly constructed. Rather, they are implemented as fast implicit analysis and synthesis operators. The complexity of these operators for the translation invariant wavelets $D_{\text {wav }}$, local DCT $D_{\text {dct }}$ and curvelets $D_{\text {curv }}$ is $O(N \log N)$ operations. For local learned dictionaries, the matrices $D_{s} \in \mathbb{R}^{n \times m_{s}}$ are explicitly constructed, but their size is much smaller than that of global dictionaries.

The number of required iterations depends on the tolerance $\eta_{\text {coef }}$ used and on the algorithm used to perform the minimization. Nesterov multi-step descent [38, 39] enjoys a fast decay of the $\ell^{1}$-regularized objective which is $O\left(1 / \ell^{2}\right)$. this much faster than the the convergence rate of iterative soft thresholding which is only $O(1 / \ell)$. In practice, Nesterov multi-step scheme performs well.

Each iteration of step 2 necessitates to reconstruct $\hat{u}_{s}$ for each $s$, which can be typically achieved in $O(N)$ or $O(N \log N)$ operations for a global dictionary, and $O\left(m_{s} n N / \Delta^{2}\right)$ operations for a local dictionary. Then the linear system (4.6) must be solved. For separation and inpainting problems, this step is fast and costs at most $O(N)$ for each $s$. The complexity of each iteration of the projected gradient descent 
of step 3 is similar to the complexity of Step 2.

5. Numerical Examples. Throughout all the numerical examples, we use patches of width $\tau=10$ pixels for the local dictionaries, with an overlap $\Delta=\tau / 2$. For all experiments, we artificially add a Gaussian white noise $\varepsilon$ of standard deviation $\sigma /\|f\|_{\infty}=0.03$.

Selecting optimal values for $\mu$ and $\lambda$ is a difficult task. We set $\mu=\sigma^{2} / 30$, that has been shown by Aharon and Elad $[19,1]$ to be a good choice for denoising applications. The parameter $\lambda$ is adapted to the noise level so that the residual satisfies $\left\|f-\mathcal{K} \sum_{s} u_{s}^{\star}\right\| \approx \sqrt{N} \sigma$, which works well for separation and denoising applications.
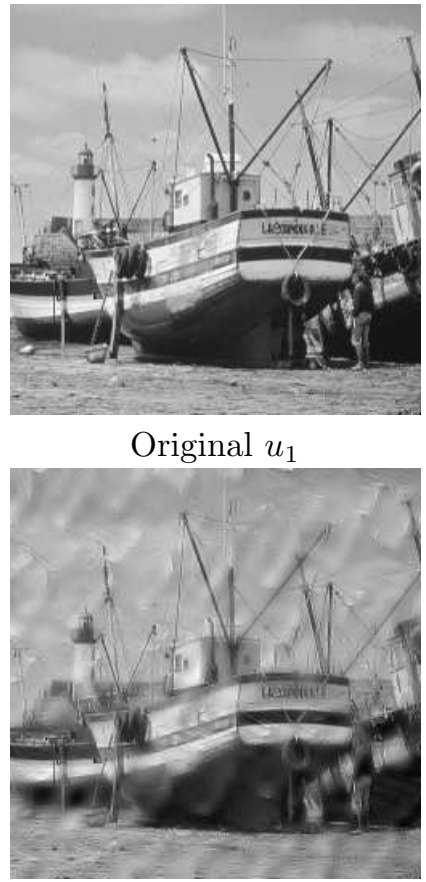

Adaptive MCA $u_{1}^{\star}$

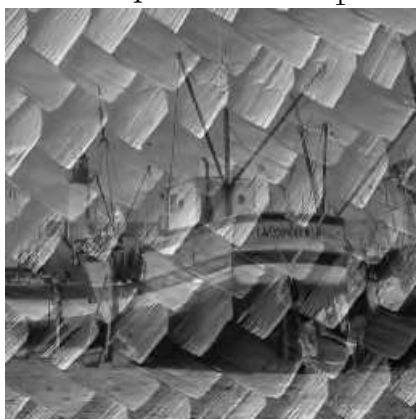

$u_{1}^{\star}$

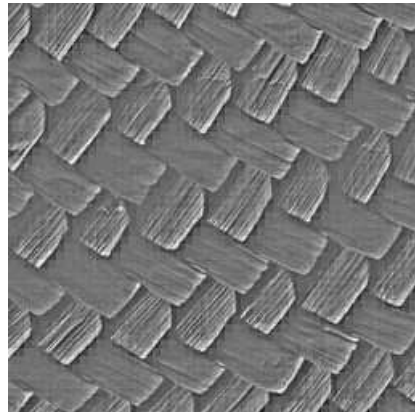

Original $u_{2}$

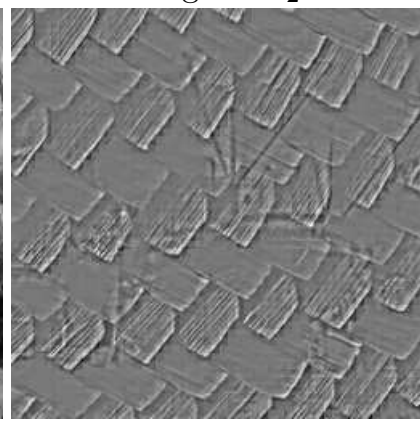

Adaptive MCA $u_{2}^{\star}$

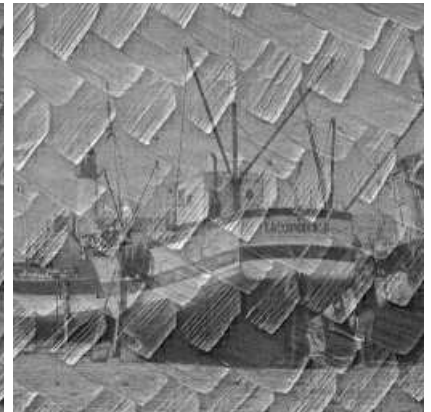

Mixture $f=u_{1}+u_{2}+\varepsilon$

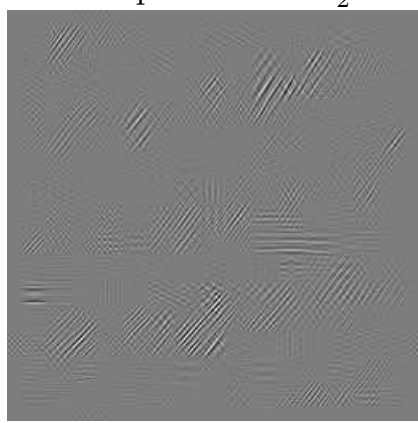

MCA $u_{2}^{\star}$

FIG. 5.1. Top row: original component to recover and observed mixture. Middle row: separation using adaptive MCA with a wavelet dictionary and an adapted local dictionary. Bottom row: separation using MCA with a wavelet and a local DCT dictionary.

5.1. Image Decomposition. We recall that the image decomposition problem corresponds to $\mathcal{K}=\operatorname{Id}_{N}$ in (4.1). One thus looks for an approximate decomposition 
$f \approx \sum_{s} u_{s}$.

Image decomposition with a single adapted layer. We perform two experiments to study the behavior of our algorithm with a fixed dictionary $D_{1}$ to capture the sketchy part of the image, and an adapted local dictionary $D_{2}$ to capture an additional homogeneous texture. When only $\left|\Lambda^{\mathrm{L}}\right|=1$ adapted layer is computed, we found that the obtained results depend only slightly on the initialization. In this case, $D_{2}$ is initialized with random patches extracted from the observations $f$.

Figure 5.1, second row, depicts the results of a first separation experiments where $D_{1}=D_{\text {wav }}$ is a fixed redundant wavelet tight frame and $D_{2}$ is an adapted dictionary. The adaptive layer is able to capture the fine scale details of the texture. Figure 5.1, third row, shows the results where $D_{2}=D_{\text {dct }}$ is a fixed local DCT tight frame. This clearly demonstrates that the local DCT is not able to capture efficiently the details of the texture, and the usefulness of the adaptivity in the separation process.

Figure 5.2, first row, displays an example of separation where the layer $u_{1}$ has a strong cartoon morphology. We thus use a fixed curvelet dictionary $D_{1}=D_{\text {curv }}$. The second layer uses an adapted local dictionary. Figure 5.2, second row, shows the separation obtained with our adaptive MCA. Although the high pass content of the texture is well capture by the adaptive dictionary, some low pass residual content is visible in the curvelet layer, mainly because elongated curvelets atoms are coherent with some patterns of the texture.

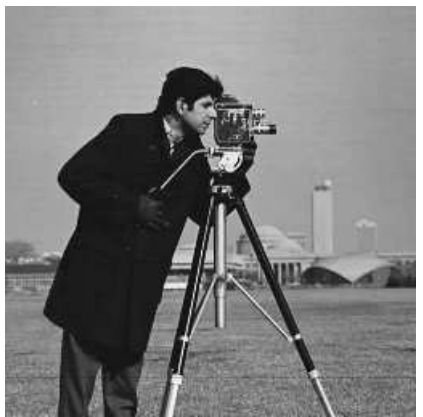

Original $u_{1}$

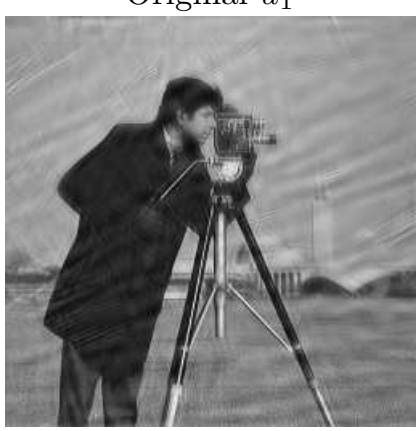

Adaptive MCA $u_{1}^{\star}$

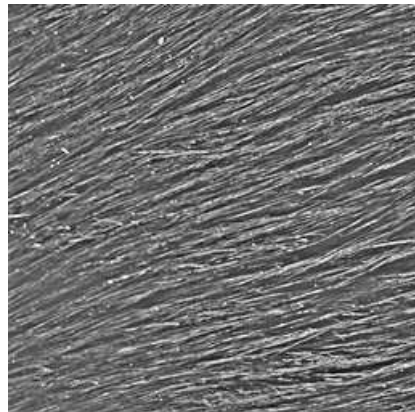

Original $u_{2}$

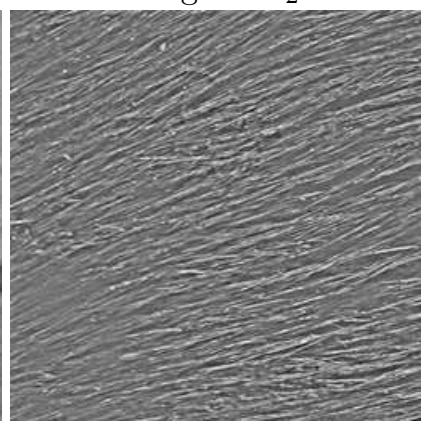

Adaptive MCA $u_{2}^{\star}$

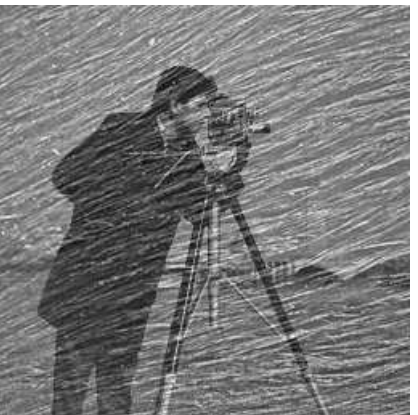

Mixture $f=u_{1}+u_{2}+\varepsilon$ tion using adaptive MCA with a curvelet dictionary and an adapted local dictionary.

Supervised separation of two textures using exemplars. In a supervised separation scenario, we consider an observed image $f=u_{1}+u_{2}+\varepsilon$ of $N=256 \times 256$ pixels, where each $u_{i}$ corresponds to a stationary texture. We also consider two exemplar textures 
$\left(\tilde{u}_{1}, \tilde{u}_{2}\right)$ of $128 \times 128$ pixels that are similar to the component to retrieve. In practice, both $u_{i}$ and $\tilde{u}_{i}$ are extracted from the same larger image, at different locations.

The local dictionaries $D_{1}, D_{2}$ are optimized during the adaptive MCA algorithm 1 . They are initialized using the exemplars $\left(\tilde{u}_{1}, \tilde{u}_{2}\right)$ by minimizing $(4.8)$ where $U_{s}$ is composed of patches extracted from the exemplars.

Figure 4.1 shows the two exemplars together with the initialized dictionaries learned from these exemplars. Figure 5.3 shows the separation obtained with our adaptive MCA, which is of high visual quality, because the two textures have a large morphological diversity.

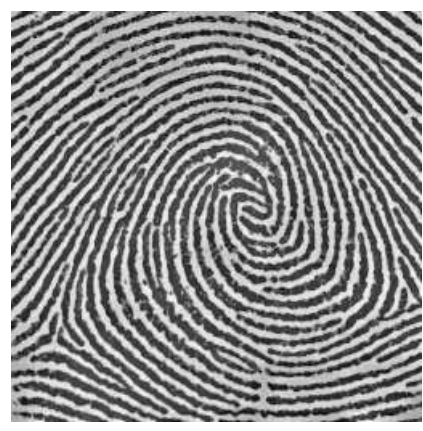

Original $u_{1}$

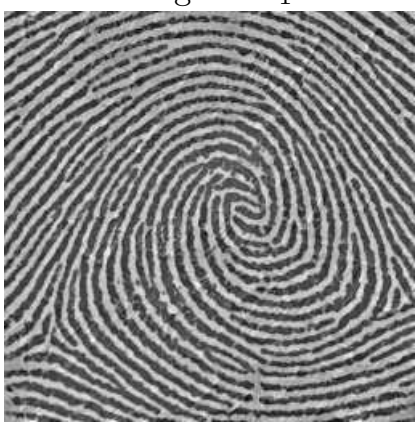

Recovered $u_{1}^{\star}$

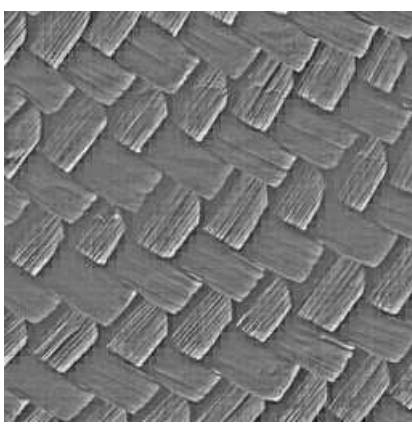

Original $u_{2}$

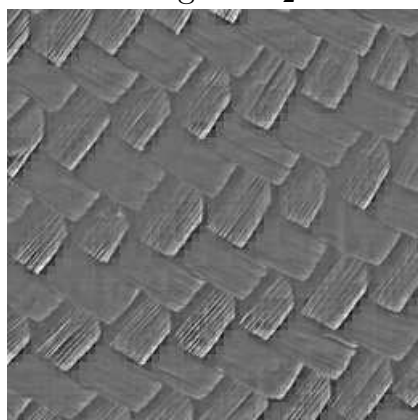

Recovered $u_{2}^{\star}$

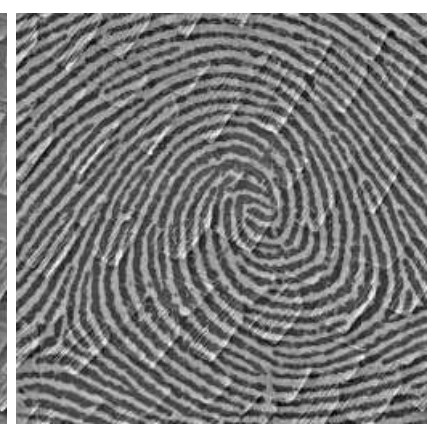

Mixture $f=u_{1}+u_{2}+\varepsilon$

Un-supervised separation. To study the ability of the adaptive MCA to automatically discriminate two complicated texture, Figure 5.4, left, shows a synthetic image obtained by linearly blending two textures $\left(\tilde{u}_{1}, \tilde{u}_{2}\right)$ as follow

$$
f[i]=\gamma[i] \tilde{u}_{1}[i]+(1-\gamma[i]) \tilde{u}_{2}[i]+\varepsilon[i]
$$

where $\gamma$ is linearly decaying from 1 to 0 along the horizontal axis.

We use two adapted dictionaries $\left(D_{1}, D_{2}\right)$ to perform the separation, and these dictionaries are initialized by extracting random patches respectively from the left and the right part of the observed image $f$.

5.2. Inpainting. Figure 5.5 depicts an example of inpainting to restore an image $f=\mathcal{K} u+\varepsilon$ where $65 \%$ of the pixels are missing. The original image $u=\sum_{s=1}^{3} u_{s}$ is a superposition of a piecewise-smooth cartoon layer, a locally oscillating texture (scarf), and a texture similar to the one in Figure 5.1. This figure compares the result of inpainting with and without an additional layer corresponding to the local learned 

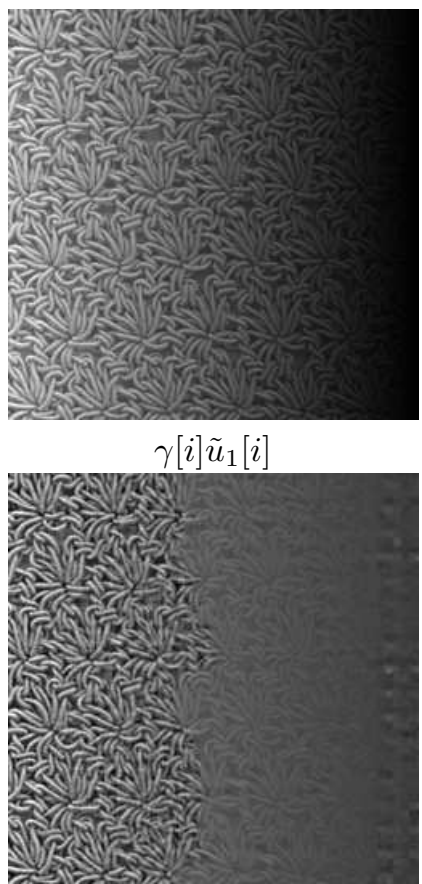

Adaptive MCA $u_{1}^{\star}$

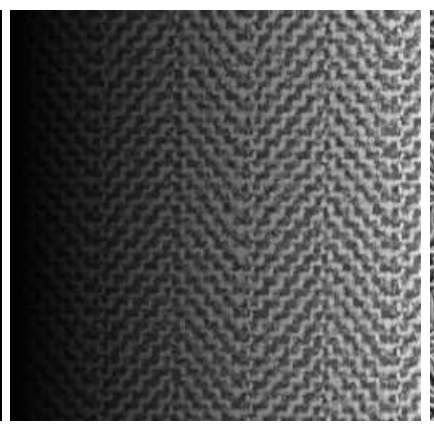

$(1-\gamma[i]) \tilde{u}_{2}[i]$

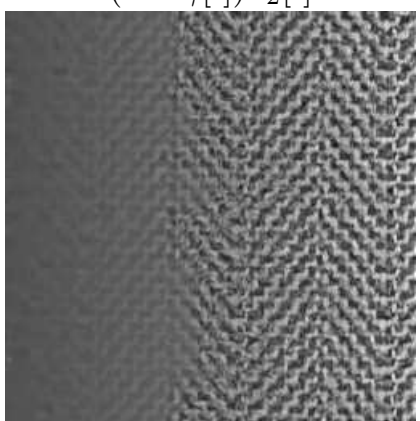

Adaptive MCA $u_{2}^{\star}$

FIG. 5.4. Example of adaptive separation using two learned dictionaries.

dictionary $D_{3}$, with redundancy $m_{3} / n=2$. In both results, the global dictionaries $D_{\text {wav }}$ and $D_{\text {dct }}$ were used. The signal to noise ratio between the original image $u$ and the recovered on $u^{\star}$ is defined as

$$
\operatorname{SNR}\left(u, u^{\star}\right)=-20 \log _{10}\left(\frac{\left\|u-u^{\star}\right\|}{\|u\|}\right) .
$$

This shows that inpainting with a learned dictionary is able to recover the missing fine scale details of the texture, which is not the case with the use of a local cosine dictionary $D_{\text {dct }}$ alone to represent the texture.

Figure 5.6 shows another example of inpainting with larger gaps. Again, the inpainting with an additional learned dictionary $D_{3}$ brings some improvement over the inpainting obtained using standard MCA with only global dictionaries, although the improvement is visually less important than with the random mask used in Figure 5.5. Our method however not only solves the inpainting problem, but also computes a visually appealing separation of the resulting inpainted image, which might be relevant for some applications in computer vision.

Conclusion. We have proposed a new adaptive method to perform structure and texture separation using both global and local dictionaries, along with an application to the inpainting problem. The main feature of the method is its ability to jointly decompose the image and learn the local dictionaries, which allows to adapt the process to the properties of the textures to be extracted. Convergence of the algorithm to a stationary point was proved. Numerical examples have shown that this adaptivity improves the efficiency and visual quality of the separation and inpainting. We have also shown that handling several learned dictionary is possible, but this requires a special care to be taken at the initialization to achieve the desired separation effect. 

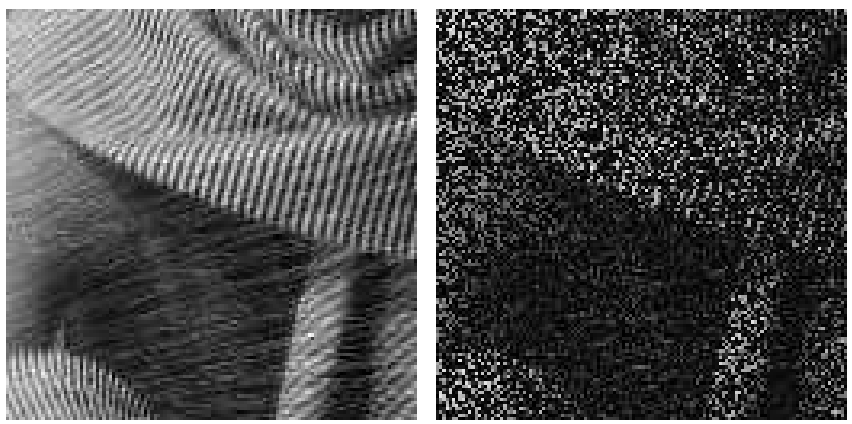

Original Image $u=\sum_{s=1}^{3} u_{s}$ Input Image $f=\mathcal{K} u+\varepsilon$
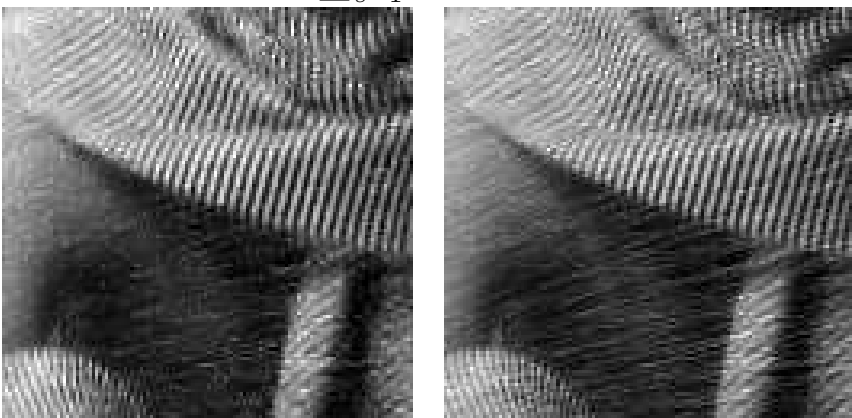

$\mathrm{MCA}, \mathrm{SNR}=14.8 \mathrm{~dB}$

Adaptive MCA, $\mathrm{SNR}=17.3 \mathrm{~dB}$

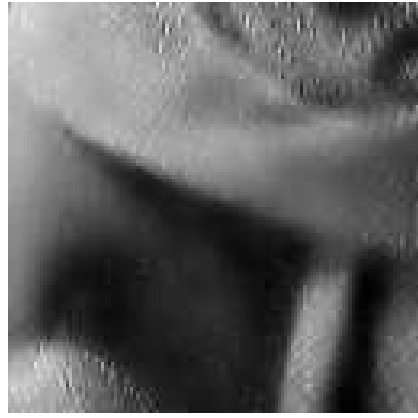

Cartoon layer $u_{1}$

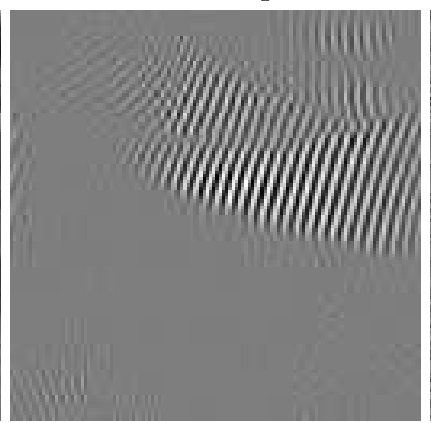

Local cosines layer $u_{2}$

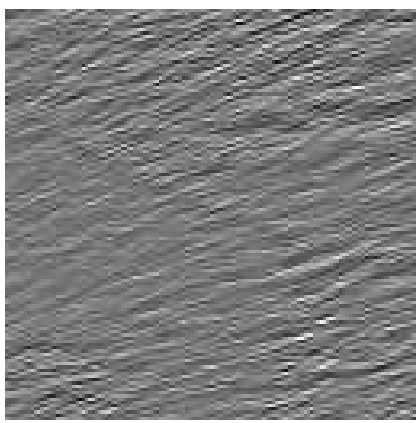

Learned dictionary $u_{3}$

FIG. 5.5. Top row: original image and masked image with 65\% randomly removed pixels. Middle row: inpainted images provided by the standard and adaptive MCA algorithms. Bottom: the three layers provided by the adaptive MCA algorithm.

\section{REFERENCES}

[1] M. Aharon, M. Elad, and A.M. Bruckstein, The K-SVD: An algorithm for designing of overcomplete dictionaries for sparse representation, IEEE Trans. On Signal Processing, 54 (2006), pp. 4311-4322.

[2] J. F. Aujol, G. Aubert, L. Blanc-Feraud, and A. Chambolle, Image decomposition into a bounded variation component and an oscillating component, Journal of Math. Im. and Vision, 22 (2005), pp. 71-88.

[3] C. Ballester, M. Bertalmio, V. Caselles, G. Sapiro, and J. Verdera, Filling-in by joint interpolation of vector fields and gray levels, IEEE Trans. Image Processing, 10 (2001), pp. 1200-1211.

[4] A. J. Bell and T. J. Sejnowski, The independent components of natural scenes are edge filters, Vision Research, (1997), pp. 3327-3338.

[5] M. Bertalmì, G. Sapiro, V. Caselles, and C. Ballester, Image inpainting, in Siggraph 2000, 2000, pp. 417-424.

[6] J. BioucAs-DiAs, Bayesian wavelet-based image deconvolution: a gem algorithm exploiting a 


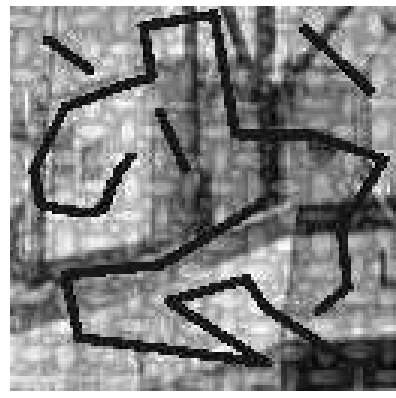

Input Image

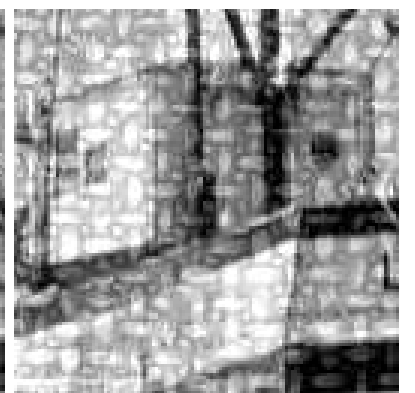

$\mathrm{MCA}, \mathrm{SNR}=16.5 \mathrm{~dB}$

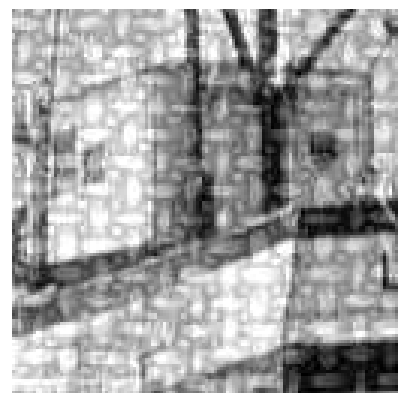

Adaptive MCA, $\mathrm{SNR}=17.8 \mathrm{~dB}$

FIG. 5.6. Image inpainting with a structured mask containing large gaps.

class of heavy-tailed priors, IEEE Transactions on Image Processing, 15 (2006), pp. 937951.

[7] J. Bobin, J-L. Starck, M.J. Fadili, and Y. Moudden, Sparsity and morphological diversity in blind source separation, IEEE Transactions on Image Processing, 16 (2007), pp. $2662-$ 2674 .

[8] J. Bobin, J.-L Starck, M. J. Fadili, Y. Moudden, and D. L. Donoho, Morphological component analysis: An adaptive thresholding strategy, IEEE Transactions on Image Processing, 16 (2007), pp. $2675-2681$.

[9] E. CANdès And D. Donoho, New tight frames of curvelets and optimal representations of objects with piecewise $C^{2}$ singularities, Comm. Pure Appl. Math., 57 (2004), pp. 219-266.

[10] V. Caselles, J. M. Morel, and C. Sbert, An axiomatic approach to image interpolation, IEEE Trans. Image Processing, 7 (1998), pp. 376-386.

[11] S. S. Chen, D.L. Donoho, and M.A. Saunders, Atomic decomposition by basis pursuit, SIAM Journal on Scientific Computing, 20 (1998), pp. 33-61.

[12] P. L. Combettes And V. R. WAJs, Gsignal recovery by proximal forward-backward splitting, SIAM Journal on Multiscale Modeling and Simulation, 4 (2005).

[13] A. Criminisi, P. PÉrez, And K. TOYama, Region filling and object removal by exemplar-based image inpainting, IEEE Transactions on Image Processing, 13 (2004), pp. 1200-1212.

[14] I. Daubechies, M. Defrise, and C. De Mol, An iterative thresholding algorithm for linear

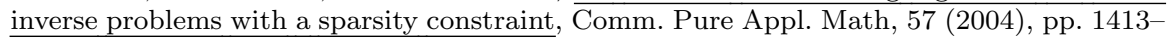
1541.

[15] L. Demanet And L. Ying, Wave atoms and sparsity of oscillatory patterns, Applied and Computational Harmonic Analysis, 23 (2007), pp. 368-387.

[16] D. Donoho, Wedgelets: Nearly-minimax estimation of edges, Ann. Statist, 27 (1999), pp. 353382.

[17] D. Donoho And I. Johnstone, Ideal spatial adaptation via wavelet shrinkage, Biometrika, 81 (1994), pp. 425-455.

[18] B. Efron, T. Hastie, I. Johnstone, and T. Tibshirani, Least angle regression, Annals of Statistics, 32 (2004), pp. 407-499.

[19] M. Elad AND M. Aharon, Image denoising via sparse and redundant representations over learned dictionaries, IEEE Trans. on Image Processing, 15 (2006), pp. 3736-3745.

[20] M. Elad, P. Milanfar, and R. Rubinstein, Analysis versus synthesis in signal priors, Inverse Problems, 23 (2007), pp. 947-968.

[21] M. Elad, J.-L Starck, D. Donoho, and P. Querre, Simultaneous cartoon and texture image inpainting using morphological component analysis (mca), Journal on Applied and Computational Harmonic Analysis, 19 (2005), pp. 340-358.

[22] K. Engan, S. O. Aase, and J. Hakon Husoy, Method of optimal directions for frame design, in Proc. ICASSP '99, Washington, DC, USA, 1999, IEEE Computer Society, pp. 2443-2446.

[23] M.J. FAdili, J.-L. Starck, And F. Murtagh, Inpainting and zooming using sparse representations, The Computer Journal (to appear), (2006).

[24] M. J. FADILI AND J.-L. STARCK, Sparse representation-based image deconvolution by iterative thresholding, in Astronomical Data Analysis IV, F. Murtagh and J.-L. Starck, eds., Marseille, France, 2006.

[25] M. Figueiredo And R. NowAK, An EM algorithm for wavelet-based image restoration, IEEE Transactions on Image Processing, 12 (2003), pp. 906-916.

[26] - A bound optimization approach to wavelet-based image deconvolution, in IEEE ICIP, 2005. 
[27] K. Kreutz-Delgado, J. F. Murray, B. D. Rao, K. Engan, T-W. Lee, and T.J. SeJNOWSKI, Dictionary learning algorithms for sparse representation, Neural Comput., 15 (2003), pp. 349-396.

[28] E. Le Pennec and S. Mallat, Bandelet Image Approximation and Compression, SIAM Multiscale Modeling and Simulation, 4 (2005), pp. 992-1039.

[29] M. S. LewiCki And T. J. Sejnowski, Learning overcomplete representations, Neural Comput., 12 (2000), pp. 337-365.

[30] L. LiEU AND L. Vese, Image restoration and decompostion via bounded total variation and negative hilbert-sobolev spaces, 2005. UCLA CAM Report 05-33.

[31] J. Mairal, F. Bach, J. Ponce, G. Sapiro, and A. Zisserman, Supervised dictionary learning, Proc. Advances Neural Information Processing Systems, (2008).

[32] J. Mairal, M. Elad, ANd G. Sapiro, Sparse representation for color image restoration, IMA Preprint Series 2139, (2006).

[33] S. Mallat, A Wavelet Tour of Signal Processing, Academic Press, San Diego, 1998.

[34] S. Mallat, Geometrical grouplets, to Appear in Applied and Computational Harmonic Analysis, (2008).

[35] S. Masnou, Disocclusion: a variational approach using level lines, IEEE Trans. Image Processing, 11 (2002), pp. 68-76.

[36] F. G. Meyer and R. R. Coifman, Brushlets: A tool for directional image analysis and image compression, Journal of Appl. and Comput. Harmonic Analysis, 5 (1997), pp. 147-187.

[37] Y. MEYER, Oscillating Patterns in Image Processing and Nonlinear Evolution Equations, American Mathematical Society, Boston, MA, USA, 2001.

[38] Y. Nesterov, Smooth minimization of non-smooth functions, Math. Program., 103 (2005), pp. $127-152$.

[39] Yu. Nesterov, Gradient methods for minimizing composite objective function, CORE Discussion Papers 2007076, Université catholique de Louvain, Center for Operations Research and Econometrics (CORE), Sept. 2007.

[40] B. A. Olshausen And D. J. Field, Emergence of simple-cell receptive-field properties by learning a sparse code for natural images, Nature, 381 (1996), pp. 607-609.

[41] M. R. Osborne, Brett Presnell, and B. A. Turlach, A new approach to variable selection in least squares problems, IMA Journal of Numerical Analysis, 20 (2000), pp. 389-403.

[42] G. PEYRÉ, Sparse modeling of textures, Journal of Mathematical Imaging and Vision, 34 (2009), pp. $17-31$.

[43] — Texture processing with grouplets, to appear in IEEE Trans. on Pattern Analysis and Matching Intelligence, (2009).

[44] G. Peyré, J. Fadili, and J.L. Starck, Learning dictionaries for geometry and texture separation, in Proceedings of SPIE Wavelets XII, SPIE, 2007.

[45] G. PEYRÉ AND S. MAllat, Orthogonal bandlet bases for geometric images approximation, Communications on Pure and Applied Mathematics, 61 (2008), pp. 1173-1212.

[46] L. I. Rudin, S. Osher, And E. FAtemi, Nonlinear total variation based noise removal algorithms, Phys. D, 60 (1992), pp. 259-268.

[47] N. SHOHAM AND M. ElAD, Alternating ksvd-denoising for texture separation, in The IEEE 25th Convention of Electrical and Electronics Engineers in Israel, IEEE Computer Society, 2008.

[48] J.-L. Starck, M. Elad, And D.L. Donoho, Redundant multiscale transforms and their application for morphological component analysis, Advances in Imaging and Electron Physics, 132 (2004).

[49] _ Image decomposition via the combination of sparse representation and a variational approach, IEEE Transaction on Image Processing, 14 (2005), pp. 1570-1582.

[50] D. Tschumperlé And R. Deriche, Vector-valued image regularization with PDEs: Acommon framework for different applications, IEEE Trans. Pattern Anal. Mach. Intell, 27 (2005), pp. 506-517.

[51] P. Tseng, Convergence of a block coordinate descent method for nondifferentiable minimization, Journal of Optimization Theory and Applications, 109 (2001), pp. 475-494.

[52] L.A. VESE AND S.J. Osher, Modeling textures with total variation minimization and oscillating patterns in image processing, Journal of Scientific Computing, 19 (2003), pp. 553-572.

[53] M. Zibulevsky and B. A. Pearlmutter, Blind source separation by sparse decomposition in a signal dictionary, Neural Computation, 13 (2001), pp. 863-882. 